\title{
Knowledge of cancer pain management among nurses in a Nigerian tertiary health institution
}

\author{
Theresa N. Elumelu, Adeniyi A. Adenipekun, Lucy O. Eriba, Bidemi I. Akinlade \\ Department of Radiotherapy, University College Hospital, Ibadan, Nigeria.
}

Correspondence: Theresa N. Elumelu. Address: Department of Radiotherapy, University College Hospital, Ibadan, Nigeria. Email: telumelu@com.ui.edu.ng

Received: February 25, 2013

DOI : $10.5430 /$ jnep.v4n4p74
Accepted: November 25, 2013 Online Published: January 19, 2014

URL: http://dx.doi.org/10.5430/jnep.v4n4p74

\section{Abstract}

Background: Pain is a symptom of both physical and psychological disorder in patients. Pain may be acute or chronic. Chronic pain can also be the consequence of progressive diseases such as cancer. Cancer pain can be defined as a complex sensation that reflects both damage to the body and the body's response to the damage. Proper pain management is crucial in the overall care of patients. The doctor-patient ratio is still low in Nigeria according to World Health Organization (WHO). 2012, we have 1:3500 doctor-patient ratio as against the standard 1:600, but that of nurse-patient is better, 1:30 as against WHO standard of 1:6, however more nurses are needed to meet the need of the rising population of Nigeria. Incidence of cancer is rising worldwide and majority of the advanced cases occur in the developing countries like Nigeria. In order to uphold the standard of cancer pain management in our patients, active participation of the nurses is essential. More importantly all health professionals need to serve as advocates for adequate cancer pain control and should ensure that pain treatment is based on ethical principles and evidence-based standards. However nurses play a critical role in effective pain management because they have more contact time with patients in any healthcare setting. This vantage position gives the nurse a unique privilege to identify patients who have pain, assess the pain and its impact on the patients and their family members, thus initiate actions to manage the pain and evaluate the effectiveness of those actions.

Aims and Objectives: This study is aimed at assessing the level of knowledge of cancer pain management among trained Nurses at the University College Hospital (UCH), Ibadan, Nigeria.

Materials and Methods: Trained nurses who consented to take part in the study were tested using self-administered questionnaire. The questionnaires consisted of 3 major sections namely (a) Demography, (b) Pain assessment, (c) Cancer Pain management. Their individual level of knowledge of cancer pain management was thereafter assessed from their response to the questionnaires.

Results: 119 Nurses completed the questionnaire. 105 (88.24\%) were double qualified, Registered Nurse (RN) and Registered Midwife (RM), while 10 (8.40\%) had only RN certificate. Approximately 21 (18\%) respondents had additional formal training in pain management. 100 (84\%) respondents gave correct definition of pain and out of these, only 2 (2\%) could give a good account on the management of cancer pain. 24 (20\%) of the respondent could use Visual Analogue Scale (VAS) for pain assessment. Only 23 (19\%) could state the relevance of opioids in cancer pain management.

Conclusion: This study revealed inadequate knowledge in cancer pain management among the nurses. To achieve holistic care for cancer patients in all health care delivery centers, additional training in pain management is required. 


\section{Key words}

Knowledge, Cancer pain management, Trained nurses

\section{I ntroduction}

Pain is an unpleasant sensory and emotional experience that is usually associated with actual or potential tissue damage ${ }^{[1]}$. Pain is a major health complaint by patients in most health care institutions. Pain management is multidisciplinary in approach but in a developing country like Nigeria, not all health professionals are knowledgeable about pain management. Nurses are major players in pain management especially in a country where we still don't have adequate number of doctors. It is estimated that Nigeria currently has a poor doctor-patient ratio of 1:3500 against the World Health Organization (WHO) standard of 1:600.This is grossly inadequate to cater for over 150 million population. In order to uphold the standard of patients' care in health care delivery services, Nurses should possess adequate knowledge to enhance their participation in the management of cancer pain in our patients. The ratio of nurses to patients of 1:30 is still better than that of doctors to patient, however WHO standard is 1nurse to 6 patients. Cancer pain has become a major health concern, because of the rising incidence of cancer, unfortunately coming in late stages in developing countries like Nigeria.

For proper management of cancer pain in patients, a care provider is expected to have ample understanding of the nature of pain syndrome and good pain assessment skills. There are two categories of pain namely nociceptive and non-nociceptive (neuropathic) pains.

Nociceptive pain refers to the response of the nervous system to tissue damage. Depending on the location and symptoms of the painful stimulus, nociceptive pain is further divided into two: somatic and viscera pain. Somatic pain is usually presented as a well-localized sharp, stabbing (knife-like) and aching; while viscera pain is difficult to localize. Somatic pain is associated with damage to the skin, muscle and connective tissues, while viscera pain is associated with cramping and dull ache ${ }^{[2]}$.

Non-nociceptive pain encompasses both the neuropathic and psychogenic pain syndromes. In both cases, nervous system responses are atypical. Non-nociceptive pain could occur without actual or obvious tissue damage.

The type of pain experienced by cancer patients is complex multi-dimensional in nature. Pain is the most feared symptom associated with cancer diseases and it is commonly experienced by over $70 \%$ of cancer patients ${ }^{[3]}$. Cancer pain is usually linked with tumor growth and the pressure it exerts on the surrounding tissues and nerves. Also, the cumulative effect of procedures undergone by cancer patients in the course of cancer management may result in pain.

There are different modalities of pain management and the outcome of each application depends largely on the skills exercised by the care giver, especially the Nurses who play major role in patient's care in most health care delivery clinics, in particular at the primary health care centers in rural areas. Somatic pain would be effectively managed with opioids medications whereas, neuropathic pain is best managed with anti-convulsant or anti-depressant drugs. To be able to participate meaningfully in pain management, trained Nurses are expected to possess adequate knowledge to handle different types of pain in patients. The role of Nurses in patient care is diverse and multi-faceted. Pain management is an integral part of patient's care and Nurses are the bed rock. . Therefore, the role of Nurses in pain management cannot be over emphasized. They are active participants in the entire clinical processes of pain management.

The processes of cancer pain management include assessment with Visual Analogue Scale (VAS) of 0 - 10 (where 0 - 3 means mild pain; 4- 7 means moderate and 8-10 means severe pain) or Verbal Rating Scale (VRS) graded as mild, moderate and severe. This is required for planning the appropriate control mechanism/medication and actual admini- 
stration of the chosen medication. The Nurses also participate in the evaluation of pain therapy, hence the need for Nurses to have good understanding of pain management.

\section{Objectives}

This study is to assess the level of competence in cancer pain management among the trained Nurses in a teaching hospital, where the best hands among Nurses are usually retained to care for ever increasing incidence of advanced cancer cases, situation in which $70 \%$ of patients present with one form of pain or the other.

\section{Methodology}

Nurses who were assigned to wards, where cancer patients were managed at the University College Hospital, Ibadan were included in the study. The objective of the study was explained to the Nurses and each was given a set of questionnaires to fill. The questionnaire consisted of 3 major sections, (a) Demography, under this heading information about additional training / qualifications were asked, (b) Pain assessment methods, with emphasis on visual analogue scale, verbal rating scale, (c) Methods of cancer pain management, questions on pharmacological and physical therapy options were asked and questions were also asked on role of opioids especially oral morphine in cancer pain. The questionnaires having about 17 stem questions have been previously pilot tested. They were made to understand that participation was voluntary. After a period of one month, out of 150 Nurses on shift duties in about five different wards, who met the criteria for selection, one hundred and nineteen (119) returned their completed questionnaires and these were analyzed.

\section{Results}

The respondents who submitted completed questionnaires were 119 Nurses. These comprised 116 (97\%) females and 3 (3\%) males. Their ages ranged from 31-60 year. Most 105 (88.24\%) of the respondents had double qualifications: registered Nurse (RN) and registered Midwife (RM). Their years of clinical nursing experience ranged from 11-20 years. All these are shown in Table 1 . There were 31 uncompleted questionnaires, all from female nurses with similar age range 29-60 year.

Table 1. The demographic data and training Information of Respondents

\begin{tabular}{ll}
\hline Parameter & Number of Respondent (\%) \\
\hline Gender status: & $3(3 \%)$ \\
Male & $116(97 \%)$ \\
Female & \\
Age (years): & $31-60$ \\
Range & $31-40$ \\
Modal age range & $11-20$ \\
Period of clinical Nursing experience (years) & \\
Acquired Nursing Certificate: & $105(88.24 \%)$ \\
$\quad$ Both certificates (RN and RM) & $10(8.40 \%)$ \\
Registered Nurse (RN) alone & $4(3.36 \%)$ \\
Registered Midwife (RM) alone & $21(17.65 \%)$ \\
Additional training (formal) in pain management & $105(88.24 \%)$ \\
Acquired Training: & $21(17.64 \%)$ \\
General Nursing and midwifery & \\
(Additional training) Pain management
\end{tabular}


Table 2 showed that in addition to general Nursing certificate acquired by all the respondents, 21 (17.65\%) of them had additional training in cancer pain management.

Table 2. Response to Cancer pain assessment/management

\begin{tabular}{|c|c|}
\hline Parameter & Number of Respondent (\%) \\
\hline \multicolumn{2}{|l|}{ Definition of terms: } \\
\hline Correct definition of pain & $100(84.03 \%)$ \\
\hline \multicolumn{2}{|l|}{ Definition of “Total” pain: } \\
\hline Correct definition of total pain & $33(27.7 \%)$ \\
\hline Limited idea about total pain & $86(72 \%)$ \\
\hline \multicolumn{2}{|l|}{ Pain assessment: } \\
\hline By observation & $55(46.22 \%)$ \\
\hline The use of pain rating scales & $24(20.17 \%)$ \\
\hline Limited idea & $40(33.61 \%)$ \\
\hline \multicolumn{2}{|l|}{ Pain management: } \\
\hline Adequate knowledge & $2 / 100(2 \%)$ \\
\hline Limited idea & $98 / 100(98 \%)$ \\
\hline \multicolumn{2}{|l|}{ Relevance of opioids (Morphine) in pain management: } \\
\hline Knowledgeable & $23(19.3 \%)$ \\
\hline No idea & $96(80.7 \%)$ \\
\hline \multicolumn{2}{|l|}{ Frequency of oral morphine administration: } \\
\hline 4 hourly & $41(34.45 \%)$ \\
\hline Limited idea & $78(65.54 \%)$ \\
\hline \multicolumn{2}{|l|}{ Preferred route of opioid (Morphine) administration: } \\
\hline Oral & $73(61.34 \%)$ \\
\hline Other routes/No idea & $46(38.65 \%)$ \\
\hline \multicolumn{2}{|l|}{ Other treatment options for cancer pain: } \\
\hline Combination of palliative, psychotherapy and radiotherapy & $7(5.88 \%)$ \\
\hline Other options & $37(31.09 \%)$ \\
\hline Limited idea/Not sure & $75(63.02 \%)$ \\
\hline
\end{tabular}

When asked to define pain, 100 (84\%) respondents got it right and 19 (16\%) had no idea. Out of these hundred respondents who defined pain correctly, only $2 \%$ actually gave detail account on how pain could be managed.

When asked to define total pain, just 33 (27.7\%) could give satisfactory definition of "total” pain, while 86 (72\%) came up with varied definitions.

On pain assessment procedure, 55 (46.2\%) of the respondents said pain should be assessed by physical observation of patients; 24 (20\%) would use pain rating scale; and 40 (33.6\%) did not know what to do. 90 (75.6\%) of the respondents were able to classify pain correctly.

When asked about the relevance of opioids (Morphine) in the management of cancer pain, 96 (80.7\%) of the respondents had no idea on the relevance of opioids as potent analgesics for severe pain management. 41(34.5\%) of the respondents knew the interval for oral administration morphine to be 4 hourly and 78 (66\%) did not know the appropriate duration. On the preferred route of morphine administration and its side effect on patient, only73 (61\%) knew the preferred route of administering and $89 \%$ knew its side effects on patients. 
$30 \%$ of the nurses said opioids should not be used freely because they are addictive, expensive, too strong and unavailable, and they would not prescribe morphine for patients in pain.

When asked about cancer pain management, 75 (63\%) had no idea; 37 (31\%) gave other options and only 7 (5.88\%) stated a combination of Palliative, Psychotherapy and Radiotherapy could be used to manage cancer pain.

\section{Discussion}

Total pain is defined as the physical, social, emotional and spiritual pain a patient goes through. In this study, only 33 (27.7\%) of the participants could define total pain. It is the knowledge of the nature of pain, acquired by the care provider that would guarantee effective management of pain.

The best approach to cancer pain management is through both pharmacologic and non-pharmacologic modalities. In this study, only $24 \%$ of the respondents got it right.

According to World Health Organization (WHO), analgesic management of pain which is a three-step approach, provides an effective treatment of pain at various levels of severity ${ }^{[4,5]}$. This approach involves the use of non-opioid medications such as acetaminophen at the first level. If the patient's pain still persists after medication, a weak opioid (also referred to as 'opioids for mild to moderate pain' -Revised EAPC recommendation on opioids in cancer pain) is initiated in addition to the previous medications at the second level. For treatment of worsening pain, a weak opioid medication is discontinued and replaced with a strong opioid at the third level ${ }^{[6]}$ ('opioid for moderate to severe pain' - Revised EAPC recommendation on opioids in cancer pain). Some physicians using the WHO guidelines are usually faced with the problem of deciding which medication, especially the opioids, to start with and at what dosage when changing to strong opioids. Once pain control becomes inadequate, the physician can use a higher tier for pain management ${ }^{[7]}$

Following the advances in pharmacogenomics, it is increasingly clear that genetics play a vital role in patient's sensitivity to analgesic. If a patient does not respond to a particular pain relief agent, the current medical regimen should be increased to maximum tolerance dose or switched to a different agent. About $70 \%$ to $85 \%$ of patients having pain related to cancer are managed with WHO pain management approach ${ }^{[8 ; 9]}$.

Another treatment modality that could be administered for cancer pain management is the adjuvant therapy ${ }^{[10,11]}$. This is initiated at any point of WHO analgesic regime as supplement. Adjuvant therapy involves the use of medication tailored towards specific causes of nociceptive pain, neuropathic pain, and reduction of opioid side effects.

Neuropathic pain is generally not responsive to opioid medications, unless significantly high doses are used; drugs shown to be effective in the management of neuropathic symptoms are Gabapentin, Carbamazepine and Pregabalin ${ }^{[12]}$. These are classified as anti-convulsants. Both Gabapentin and Pregabalin are excreted by renal route and have minimal drug - drug interactions. These make them effective first line treatment for neuropathic pain ${ }^{[13]}$.

Another category of medications that could be used to stabilize neurons involved in neuropathic pain is the anti-depressants. Low doses of tricyclic anti-depressants such as Amitriptyline, has been shown to be effective for pain relief ${ }^{[14]}$. Other medications that are seldom prescribed for neuropathic pain are serotonin re-uptake inhibitors and newer classes of anti-depressants such as selective serotonin inhibitors. Since most patients with neuropathic pain often have depressive or anxiety symptoms, anti-depressant medication with its potentials to treat both conditions, has shown improved efficacy in the treatment of neuropathic pain.

Inadequate knowledge of analgesic drugs administered to patients is one of the commonly cited reasons for under treatment of pain ${ }^{[15]}$. In this study, $70 \%$ of the respondents have heard about opioids, but never knew how it should be administered. This is consistent with previous studies where findings showed that there are significant deficiencies on the 
general application of opioid drug. Opioid analgesics are the main drugs used to control moderate to severe pain, particularly in cancer patients. Despite their recognized effectiveness, opioids often are not freely available because of restrictive laws based on fear of misuse and abuse. Rajagopal and his colleagues from Calicut, India, followed 1723 patients who were being treated for cancer pain with oral morphine on an outpatient, homecare basis. Over the 2 years of the study, the investigators did not identify a single instance of misuse or diversion ${ }^{[16]}$.

Nursing staff occupy a central position in the management of patients' pain and most often, they are the first to identify the problem of uncontrolled pain. In this study, only $20 \%$ of the Nurses interviewed knew a rating scale should be used to assess pain in patients before commencement of appropriate management.

In a study by Bernardi $\mathrm{M}$ et al on a national survey of Italian hospice nurses, analysis of items showed that more than $30 \%$ of hospice nurses underestimated the patients' pain and they did not treat the pain in the correct way; they had an incorrect self-evaluation about their pain management knowledge. Results from stepwise regression showed that nurses with higher correct answer scores had attended more courses on pain education ${ }^{[17]}$

The opinion of majority of the nursing staff is that analgesic administration should be based on patient's request and this showed that most trained Nurse-midwives do not recognize the concept of steady state analgesic, which ensures that around-the-clock dosing regimen, is administered to patient. The basis of this concept is that inability of patient to express pain or lodge a pain complaint does not mean lack of pain in that patient.

The fear of addiction expressed by some 36 (30\%) Nurses in this study as the reason why they would not freely administer opioids to manage cancer pain remains a considerable concern and an impediment to effective use of narcotic analgesics. A similar feeling was expressed by $69 \%$ of the respondents in Cohen's study ${ }^{[18]}$. Similarly in a study by Schmidt et al analysis of questionnaires from respondents who were pediatric nurses indicated that they cared for cancer patients regularly but had poor understanding of general principles of pain management for the cancer patient. Nurses had exaggerated concerns regarding the risk of addiction and respiratory depression associated with narcotic analgesics ${ }^{[19]}$.

In this study, only $34 \%$ of the respondents knew appropriate duration of administration of oral liquid morphine while the majority did not know. The experience was the same in the study conducted at the University of Ilorin Teaching Hospital, Nigeria, where a significant proportion of respondents did not know the appropriate duration of administering opioids ${ }^{[20]}$.

Most of the respondents 81 (68\%) in this study did not know any other method for cancer pain control apart from the use of analgesic. This means that they were not aware of non-pharmacological means of pain management.

\section{Conclusion}

The findings in this study showed that the knowledge of cancer pain assessment and management in Nurses, charged with the responsibility of caring for these patients in various wards at the University College Hospital, Ibadan, is insufficient. This is traceable to the fact that most of them did not have formal training in pain management either at the local or International level. Many of them are familiar with oral route of administration of opioids but the logistics of administration is not clear to them. Also, many of them did not know how to assess cancer pain nor the modalities of treatment to be used.

\section{Recommendations}

In order to improve the Nursing care of cancer patients in pains, frequent formal training and re-training in pain management should be included in their clinical practice. Make pain assessment a part of vital signs to be monitored. Participation in workshop on various types of pain and its management should be part of the promotion exercise for nursing staff. In 
addition to these, pain management should be incorporated into the curriculum of Schools of Nursing and all post basic Nursing Schools so as to produce future Nurses with appreciable knowledge of cancer pain management.

It is also recommended that practitioners in Nigeria engage with government authorities to increase awareness of the medical need for opioids, emphasizing that their lack of availability is causing severe distress and suffering among cancer patients.

\section{Acknowledgement}

Fifth Bristol Opioid Conference In association with the EAPC and EPCRC, February, 2010.

\section{References}

[1] Classification of Chronic Pain, Second Edition, IASP Task Force on Taxonomy, edited by H.

[2] Merskey and N. Bogduk, IASP Press, Seattle; 1994; 209-14.

[3] Besson JM, Chaouch A. Peripheral and spinal mechanisms of nociception Physiol. 2005; 186.

[4] Bonica JJ. Treatment of cancer pain: current status and future needs. In: Fields, H.L. et.al. ed. Advances in pain research and therapy. 1985. Vol. 9. New York, Raven Press. 589-616.

[5] Jadad AR, Browman GP. The WHO analgesic ladder for cancer pain. WHO. 1995; 274: 1870-1873.

[6] Ventafridda V, Saita L, Ripamonti C. et al. WHO guidelines for the use of analgesics in cancer pain. Int. Jour. Tissue React. 1985; 7: 93-96.

[7] Azevedo SL, Ferreira K, Kimula M, Jacobsen TM. The WHO analgesic ladder for cancer pain control, twenty years of use. How much pain relief does one get from using it? Support Care Cancer. 2006.

[8] World Health Organization. WHO guidelines cancer pain relief, 2nd ed. 1996. Geneva: World Health Organization.

[9] Palmer SN, Giesecke NM, Body SC. et al. Pharmacogenetics of anesthetic agents. Anesthesiology. 2005; 102: 663-671. http://dx.doi.org/10.1097/00000542-200503000-00028

[10] Stamer UM, Bayerer B, Stuber F. Genetics and variability in opioid response. Eur J Pain. 2005; 9: 101-105. http://dx.doi.org/10.1016/j.ejpain.2004.05.008

[11] Ventafridda V, Saita L, Ripamonti C. et al. WHO guidelines for the use of analgesics in cancer pain. Int J Tissue React. 1985 ; 7: 93 $-96$.

[12] Zech DF, Grond S, Lynch J, et al. Validation of World Health Organization Guidelines for cancer pain relief: a 10-year prospective study. Pain. 1995; 63: 65-67. http://dx.doi.org/10.1016/0304-3959(95)00017-M

[13] Milch RA. Neuropathic pain: implications for the surgeon. Surg Clin North Am. 2005; 85: 225-236. http://dx.doi.org/10.1016/j.suc.2004.12.001

[14] Irving GA .Contemporary assessment and management of neuropathic pain. Neurology 2005; 64 (suppl 12 \& 13): $21-27$.

[15] Hainline B. Chronic pain: physiological, diagnostic and management considerations. Psychiatr. Clin North Am. 2005; $28: 713-735$. Cancer Medicine 4th Edition, page 1363- 1864.

[16] Rajagopal MR, Joranson DE, Gilson AM. Medical use, misuse and diversion of opioids in India. Lancet. 2001; 358: 139-43. http://dx.doi.org/10.1016/S0140-6736(01)05322-3

[17] Bernardi M, Catania G, Knowledge and attitudes about cancer pain management: a national survey of Italian hospice nurses. G cancer Nurs. 2007 Mar-Apr; 30(2): E20-6.

[18] Cohen F. L. Post surgical pain relief: Patients’ status and nurses’ medication pain. 1980; 9: 265-274.

[19] Schmidt K, Eland J, Weiler K. J Pediatr Oncol Nurs. Pediatric cancer pain management: a survey of nurses' knowledge. 1994 Jan; 11(1): 4-12; discussion 13.

[20] Soyanwo O. A post operative pain control prescripts pattern and patients experience. West African Journal Med. 1999; 18: 207-210. 